\section{Common errors in the treatment of intra-abdominal infections: the irrational use of antimicrobial agents}

\author{
Belinda De Simone, ${ }^{1}$ Fausto Catena, ${ }^{1}$ \\ Massimo Sartelli, ${ }^{2}$ Salomone Di Saverio, ${ }^{3}$ \\ Federico Coccolini, ${ }^{4}$ Arianna Heyer, ${ }^{5}$ \\ Rodolfo Catena, ${ }^{6}$ Luca Ansaloni ${ }^{4}$ \\ 'Department of Emergency and Trauma \\ Surgery, University Hospital of Parma, \\ Parma; 'Department of Emergency and \\ General Surgery, Macerata Hospital, \\ Macerata; ${ }^{3}$ Department of General \\ Surgery, Maggiore Hospital of Bologna, \\ Bologna; ${ }^{4}$ Department of General and \\ Emergency Surgery, Papa XXIII Hospital, \\ Bergamo, Italy; ${ }^{5}$ Department of Medical \\ Sciences, University of California, \\ Berkeley, CA, USA; 'Said Business \\ School, University of Oxford, Oxford, UK
}

\begin{abstract}
Antimicrobial resistance (AR) is a global, emergent problem because an increasing numbers of serious community acquired and nosocomial infections are caused by resistant bacterial pathogens. It is a direct consequence of the excessive and irrational use of antibiotics. The use of antimicrobial agents - aimed to decrease morbidity and mortality rate related to intra-abdominal infections - is very high, often improper, in the Departments of General and Emergency Surgery and Intensive Cure Units. Source control and empiric antibiotic therapy have to be administrated as early as possible to decrease high mortality rates in patients with severe sepsis or septic shock and, in this, the general surgeon has a crucial role. Proper antimicrobial stewardship in selecting an appropriate antibiotic and optimizing its dose and duration to cure intraabdominal infections may prevent the emergence of AR and decrease costs for antibiotics.
\end{abstract}

\section{Introduction}

Antibiotics are the only drugs available to fight against infections. Their frequent, and often improper, use is seen in the department of general and emergency surgery and in the Intensive Cure Units (ICU) to decrease morbidity and mortality rate related to intraabdominal infections (IAIs). Antimicrobial resistance (AR) is a direct consequence of the excessive and irrational use of antibiotics.
More specifically, this results from the incorrect indication, spectrum, route, dose or duration of therapy regarding antimicrobials.

AR is a global, emergent problem because an increasing number of serious community acquired and nosocomial infections are caused by resistant bacterial pathogens. AR affects all major classes of antimicrobial agents, often involving multiple classes and resulting in complex resistance phenotypes for which only a very limited number of drugs remain active, leading to increased costs and deaths.

In the United States, antibiotic-resistant infections are associated with 23,000 deaths and 2 million illnesses per year, with estimated excess direct healthcare costs of $\$ 20$ and $\$ 35$ billion in lost productivity, respectively. Resistant organisms can increase patientrelated prescribing costs by $\$ 8000$ to $\$ 30,000$. $^{1}$

Italy has the highest prevalence of $\mathrm{AR}$ among European countries, in particular carbapenem-resistant Klebsiella pneumonia, which reached $34.3 \%$ in $2013 .{ }^{2}$

The World Health Organization (WHO) is highly active in the development of measures to contain the spreading of AR, providing a framework of interventions to slow the emergence and reduce the spread of antimicrobialresistant microorganisms, with the aim of optimizing appropriate antibiotic treatment, but a global collective action is necessary to control this phenomenon. In the last years, many antibiotic stewardship programs has been proposed with the objective to ensure effective treatment for patients with bacterial infection and to support professionals and patients to reduce unnecessary use and minimize collateral effects and excess cost.

\section{Opinion Report}

\section{Etiology and antibiotic resistance}

The etiology of IAIs is often polymicrobial and usually includes organisms derived from the gut microbiota. In community-acquired IAIs enterobacteria predominate (mostly Escherichia coli) in combination with anaerobes (mostly Bacteroides fragilis). In nosocomial IAIs, which can complicate abdominal surgery, pathogens such as Pseudomonas aeruginosa, Acinetobacter baumannii, Staphylococcus aureus, Enterococcus spp. and Candida spp. are often involved. ${ }^{3,4}$

Infections originating from the stomach, duodenum, bile ducts, or proximal small intestine are mostly caused by Gram-positive and Gram-negative facultative aerobic bacteria. Infections originating from the ileum are mainly caused by facultative aerobic and obligate aerobic Gram-negative bacteria (such as e.g. Bacteroides fragilis). In the case that the colon is the organ of origin, facultative and
Correspondence: Belinda De Simone, Department of Emergency and Trauma Surgery, University Hospital of Parma, via Gramsci 15, 43100 Parma, Italy.

Tel: +39.320 .0771984 .

E-mail: desimone.belinda@gmail.com

Contributions: BDS collected data and wrote the manuscript; FC and MS read and revised the final manuscript. All the authors read and approved the manuscript.

Conflict of interest: the authors declare no potential conflict of interest.

Key words: Antibiotic resistance; Intra-abdominal infections; De-escalation strategy; Source control; Antibiotic therapy.

Received for publication: 24 May 2016. Accepted for publication: 6 June 2016.

This work is licensed under a Creative Commons Attribution 4.0 License (by-nc 4.0).

(C) Copyright B. De Simone et al., 2016

Licensee PAGEPress, Italy

Emergency Care Journal 2016; 12:6023

doi:10.4081/ecj.2016.6023

obligate anaerobic microbes are predominant (often enterococci, most often $E$. coli).,3

The WHO global report describes the majority of world regions with over $50 \%$ resistance of Escherichia coli (E. coli) and Klebsiella Pneumoniae to third generation cephalosporins and fluoroquinolones, associated with the increasing prevalence of carbapenem-resistant organisms, and other multi-resistant strains such as Methicillin resistant Staphylococcus aureus (MRSA) as well as extended-spectrum beta-lactamase (ESBL) producers. ${ }^{5}$

The AR situation in Europe displays large variations depending on the bacterium, antimicrobial group and geographical region. Over the 2011-2014, resistance to third generation cephalosporins in Klebsiella pneumoniae and Escherichia coli increased significantly. ${ }^{6}$

According to data reported by the European Antimicrobial Resistance Surveillance Network (EARS-Net) in 2014, a large proportion of the isolates resistant to third-generation cephalosporins produced an ESBL. Thirdgeneration cephalosporin resistance in combination with fluoroquinolone and aminoglycoside resistance increased between 2011 and 2014 for both $E$. coli and $K$. pneumoniae. The European Union/European Economic Area (EU/EEA) population-weighted mean for carbapenem resistance in 2014 was $7.3 \%$ for $K$. pneumoniae (resistance percentages ranged between zero and $62.3 \%$ ); carbapenem resistance remained very low in $E$. coli $(0.1 \%)$. For $P$. aeruginosa, the EU/EEA population-weighted 
mean percentage for carbapenem resistance increased significantly between 2011 and 2014. Acinetobacter spp. is the bacterium for which the highest carbapenem resistance levels were reported. For meticillin resistant Staphylococcus aureus (MRSA), the EU/EEA population-weighted mean percentage has decreased over the last four years, from $18.6 \%$ in 2011 to $17.4 \%$ in 2014 . $^{6}$

\section{The crucial role of the surgeon in the management of intra-abdomi- nal infections}

Inappropriate antibiotic use is frequent in surgical units and surgeons have an important role in the early diagnosis and management of IAIs: improper initial empiric antibiotic therapy is associated with greater morbidity.

IAIs include several different pathological conditions ranging from complicated appendicitis to stercoral peritonitis; they can be classified in uncomplicated IAI, when the infection involves a single organ and does not spread to the peritoneum; and complicated IAI, when the infectious process proceeds beyond a single organ, causing either localized or diffuse peritonitis.

Some authors prefer referring to the classification of peritonitis, which is the most frequent IAIs, into 3 different forms: ${ }^{3}$ primary peritonitis, that is hematogenous peritonitis in children, spontaneous bacterial peritonitis in adults, tuberculous peritonitis, usually in the setting of an immunocompromised state of the patient; secondary peritonitis, that is related to a pathologic process in a visceral organ, such as perforation or trauma, including iatrogenic trauma, that requires surgical or interventional treatment (it can be classified as community- and hospital-acquired-postoperative peritoniti); tertiary peritonitis, that is persistent or recurrent infection after adequate initial therapy.

According to the etiology, IAIs can be divided in 3 subgroups: i) community acquired extrabiliary infections, that include gastro-duodenal perforations, acute appendicitis, acute diverticulitis, large bowel perforation; ii) community acquired biliary infections, that include acute cholecystitis and cholangitis; iii) hospital acquired infections (postoperative and non postoperative peritonitis). ${ }^{7}$

Early control of the septic source is mandatory. Patients with uncomplicated IAIs can be treated with either surgical resection or antibiotics; when the infection is effectively resolved by surgical excision, 24-48 short therapy is sufficient. The treatment of complicated IAI provides source control surgery associated with antibiotic therapy. Stratification of the patient's risk is essential in order to optimize the treatment plan. ${ }^{8}$ According to the severity of the clinical presentation, IAIs can be divided into mild-moderate and severe; severe IAIs are associated with progressive multiple organ dysfunction, prolonged hospitalization, and high mortality. ${ }^{9}$

In the Infectious Diseases Society of America (IDSA) guidelines, patients with IAIs are defined at high risk when there are a range of reasons for increased rates of treatment failure, in addition to a higher severity of infection, particularly patients with an anatomically unfavorable infection or a health care associated infection. ${ }^{10}$

Factors predicting failure of source control for IAIs are: delay in the initial intervention ( $>24 \mathrm{~h}$ ); high severity of illness (APACHE II score $>=15$ ); advanced age; comorbidity and degree of organ dysfunction; low albumin level; poor nutritional status; degree of peritoneal involvement or diffuse peritonitis; inability to achieve adequate debridement or control of drainage; presence of malignancy. ${ }^{8}$

Sepsis is the unfavorable evolutive step of the IAIs; it is a complex, multifactorial, syndrome that can progress to condition of varying severity; there is an increased risk of death as patients' transition from sepsis to severe sepsis and septic shock. Patients affected by septic shock require early admission in ICU to administrate resuscitation, source control and large spectrum antimicrobial therapy. Mortality for sepsis in critically ill patients can reach 50\%; according to the CIAOW Study data, mortality for sepsis is 10.5 and $29.8 \%$ of surgical patients were admitted to the ICU in the early recovery phase after abdominal surgery. ${ }^{4}$

Time to initiation of antibiotic treatment is the single strongest predictor of outcome. Each hour's delay increases mortality by $7.6 \%$, over the first 6 hours. Septic shock and severe sepsis upon hospital admission were both predictive of patient mortality. Sepsis increases patient-related costs six-fold. ${ }^{1}$ In the literature, several criteria were found to be independent variables predictive of mortality, including patient age, the presence of small bowel perforation, a delayed initial intervention (a delay exceeding 24 hours), ICU admission and patient immunosuppression.-10

The surgeon has the important role to prevent the progression from sepsis to shock septic with his early treatment decisions. Sartelli highlights the importance of surgeons using a scoring system to reduce the time in evaluating prognoses, assessing the severity of the complicated IAIs and decision-making regarding the aggressiveness of ensuing treatment. To stress his point, Sartelli carried out a multicenter international observational study to validate the World Society Emergency Surgeons (WSES) Sepsis Severity Score (SSS), a practical clinical severity score for patients with complicated IAIs (Table 1). Data reported confirmed that the WSES SSS shows high sensitivity, specificity and likelihood ratio, leading to the conclusion that it may help in reaching a clinical decision in the management of patients affected by IAIs. ${ }^{8}$

\section{Source control and early empiric antibiotic therapy}

Once the diagnosis of IAI is suspected or confirmed by clinical, biological and radiological investigations, it is mandatory to begin, as soon as possible, the empiric antimicrobial therapy and simultaneously obtain source control, after the diagnosis of postoperative peritonitis. $^{7}$

The Complicated Intra-Abdominal infection Observational Worldwide (CIAOW) study proposed by the WSES, with the aim to describe the clinical, microbiological and management related profiles of both community- and health care-acquired complicated IAIs in a worldwide

Table 1. World Society Emergency Surgeons Sepsis Severity score (range 0-18) for risk's stratification of the septic patients.

\begin{tabular}{ll} 
& Score \\
Clinical condition at the admission & \\
$\quad$ Severe sepsis at admission & 3 \\
$\quad$ Septic shock at the admission & 5 \\
Setting of acquisition & \\
$\quad$ Healthcare associated infection & 2 \\
\hline $\begin{array}{l}\text { Origin of the IAIs } \\
\quad \text { Colonic non-diverticular perforated peritonitis }\end{array}$ & 2 \\
$\quad$ Small bowel perforation peritonitis & 3 \\
$\quad$ Diverticular diffuse peritonitis & 2 \\
$\quad$ Postoperative diffuse peritonitis & 2 \\
Delay in source control & 3 \\
$\quad$ Delayed initial intervention (preoperative duration of peritonitis $>24 \mathrm{~h}$ ) & 3 \\
\hline Risk factors & \\
Age $>70$ years & 2 \\
Immunosoppression & 3 \\
\hline
\end{tabular}

IAI, intra-abdominal infection. 
context, reported that the most frequent source of infection was acute appendicitis and that open appendectomy was the most common approach in the patients with complicated appendicitis. Open cholecystectomy was the most common means of treating complicated cholecystitis; Hartmann's resection was the most frequently performed procedure to address both complicated diverticulitis and non-diverticular colonic perforations worldwide. $^{4}$

Patients with septic shock and severe sepsis at admission may benefit from aggressive surgical treatment to control multiple organ dysfunction syndrome. A single surgical exploration could not be sufficient to achieve source control (SC).

The management of IAIs by SC represents a key component of success in therapy of sepsis. It includes drainage of infected fluids, debridement of infected soft tissues, removal of infected devices or foreign bodies, and finally, precise surgical techniques to correct any anatomic, iatrogenic or not derangement. SC can be achieve by re-laparotomy strategies with temporary abdomen closure or open abdomen. ${ }^{4}$

Open abdomen $(\mathrm{OA})$ procedure can be used as therapeutical option in the management of patients with severe sepsis, with the aim to control any persistent source of infection, preventing abdominal compartment syndrome and defer definitive intervention and anastomosis. It provides 3 stages: i) adequate and prompt source control; sepsis source control is based on 3 principles: drainage and lavage of the infected fluid, debridement of infected/necrotic tissue and definitive or temporary measures to correct anatomic derangements and to restore optimal function; ii) resuscitation and adequate antimicrobial therapy; iii) re-exploration of the abdomen after 24-48 hours and early and definitive closure of the abdomen. ${ }^{11}$

Early and proper empirical antibiotic therapy is fundamental to increase overall survival in septic patients; in literature, many studies confirmed that inappropriate initial empiric antibiotic therapy is associated with greater morbidity. ${ }^{12,13}$

Membrilla Fernandez et al. carried out a prospective, multi-centric (24 Spanish hospitals involved), observational study with the aim to evaluate the impact of adequate empiric antibiotic treatment associated with control of the infection, focused on the morbidity and mortality rates of patients with secondary peritonitis. The study included 362 patients (262 with community-acquired IAIs, 100 with postoperative peritonitis); the rates of therapeutic failure, re-operation, and mortality reported were 48,13 , and $8 \%$, respectively. Empiric antibiotic treatment was inappropriate in $39 \%$ of cases, and associated with a higher rate of surgical site infection (53 vs 40\%; $\mathrm{P}=0.031$ ) and death (12 vs $5 \%$; $\mathrm{P}=0.021$ ) than was observed in patients receiving appropriate initial empiric therapy. The authors concluded that inappropriate initial empiric antibiotic therapy was associated with higher rates of therapeutic failure, surgical site infection, re-operation, and death. ${ }^{14}$

The principles of empiric antibiotic treatment should be defined according to the most frequently isolated germs, always taking into consideration the local trend of antibiotic resistance and the international evidencebased guidelines.

Zhang et al. retrospectively analyzed medical records of patients with IAIs to investigate the clinical and etiological characteristics of community-acquired IAIs and hospital-acquired or nosocomial IAIs in China, to understand which antibiotics use in the initial empiric therapy; they concluded that in clinical practice, the application of international guidelines, in particular of the 2010 Infectious Diseases Society of America guidelines, is the first step of an empirical broad spectrum antimicrobial treatment, but the regimen needs to be adapted early, in accordance with regional IAIs pathogenic bacteria isolated by cultures and to their own features in drug resistance. $^{9}$

The CIAOW Study, a multi center prospective observational study which joined 68 medical institutions with a total of 1898 patients included the study, showed that the major pathogens involved in community-acquired IAIs were found to be Enterobacteriaceae (especially E. coli, Klebsiella pneumoniae, Enterobacter), Streptococcus species, and certain anaerobes (particularly B. fragilis). Extended spectrum beta lactamase (ESBL) producers were the most commonly identified drug-resistant microorganism involved in IAIs. ESBL-producing Enterobacteriaceae are becoming increasingly common in communityacquired infections. ${ }^{4}$

Pseudomonas aeruginosa (non fermenting gram negative bacterium) is one of the major nosocomial pathogens worldwide, intrinsically resistant to many drugs and it is able to become resistant to virtually any antimicrobial agent. ${ }^{4}$ Enterococci (E. faecalis and $E$. faeci$u m$ ) were the most Gram positive facultative anaerobic isolates and they were more prevalent in nosocomial infections. Enterococci infections are difficult to treat because of both intrinsic and acquired resistance to many antibiotics. Furthermore, in the last years there has been a significant increase in the incidence of invasive infections due to Candida species. ${ }^{4,12,13}$

The choice of the antimicrobial regimen depends on the source of intra-abdominal infection, the risk factors for specific microorganisms, the resistance patterns and the clinical patient's condition.
In uncomplicated IAIs, when the focus of infection is treated effectively by surgical excision of the involved tissue, the administration of antibiotics is unnecessary beyond prophylaxis. Peri-operative antibiotic prophylaxis, defined as a single administration of antibiotics shortly before a surgical intervention to reduce surgical wound infections in visceral surgery, reduces the duration of the hospital stay and health care costs. ${ }^{3}$ A prolonged prophylaxis including the postoperative period (e.g. 1-3 days postoperatively) should be avoided as it does not reduce the number of wound infections and is associated with an increased risk of AR and side effects. Prolonged peri-operative antibiotic prophylaxis is associated with an increased risk of Clostridium difficile-associated infection. ${ }^{3}$

In complicated IAIs, antimicrobial therapy is mandatory.

For patients with community acquired IAIs, the pathogens are generally predictable (Enterobacteriaceae such as E. coli, Klebsiella pneumonia, Enterobacter; Streptococcus species, and $B$. fragilis) and antimicrobial agents with narrower spectrum of activity are preferred. ${ }^{7,10,12}$

Hospital acquired infections are commonly caused by larger and more resistant flora such as ESBL producers, Pseudomonas aeruginosa, methicillin resistant Staphylococcus aureus (MRSA), Acinetobacter baumanii, vancomicyn resistant enterococcus, Klebsiella pneumoniae carbapenemases, and for these infections, complex multi-drug regimens are always recommended. ${ }^{7,10,12}$

Biliary infections are often caused by gram negative aerobes such as $E$. coli, Klebsiella pneumoniae and anaerobes as Bacteroides fragilis. ${ }^{7,10,12}$

A regimen was considered adequate when all relevant pathogens isolated in one patient were susceptible to at least one of its components. ${ }^{13}$

Diagnostic microbiology of IAIs plays a relevant role in the therapeutic strategy of every patient, in the era of the broad spread of resistant microorganisms above all in nosocomial IAIs. In community acquired infections ESBL Enterobacteriaceae, carbapenemase producing gram negatives, beta-lactam and vancomycin resistant enterococci are increasingly involved. Treatment alternatives for patients infected with bacteria resistant to both carbapenems and other key antimicrobial groups are often limited to combination therapy and to older antimicrobial agents such as polymyxins, e.g. colistin. ${ }^{12,13}$

Empirical antifungal therapy for candida species is recommended for patients with nosocomial infections. An echinocandin regimen is recommended for critical ill patients with nosocomial infections. ${ }^{?}$

Focusing on WSES guidelines for the mana- 
gement of IAIs, antimicrobial therapy for community acquired extra-biliary IAIs, in stable non critical patients without ESBL associated risk factors, provides the first line use of amoxicillin/clavulanate, or ciprofloxacin associated with metronidazole in allergic to beta lactams patients; for stable patients with risk of ESBL pathogens, ertapenem or tigecycline are indicated; for critically ill patients with no ESBL associated risk factors, piperacillin/tazobactam is the antimicrobial agent indicated, while in critically ill patients with risk of ESBL pathogens, meropenem or imipenem +/- fluconazole are indicated.

In the treatment of biliary infections, for stable patients without ESBL associated risks factors amoxicilline/clavulanate or in presence of allergic patients ciprofloxacin+metronidazole are indicated; in patients with risk of ESBL pathogens, tigecycline is indicated; for critically ill patients with biliary infections piperacillin/tazobactam is indicated in case of no risk of ESBLs, and piperacillin+tigecycline+/-fluconazole, in case of ESBL pathogens.

In the treatment of nosocomial IAIs, in stable patients piperacillin+tigecycline+fluconazole are indicated; critically ill patients have to be treated with the association of piperacillin+tigecycline+echinocandin or meropenem, imipenem, doripenem+teicoplanin+echinocandin (Table 2). ${ }^{7}$

Steinbach et al. developed a method to assess the spectrum adequacy of antimicrobial regimens in patients affected by secondary peritonitis, called battleship approach, retrospectively analyzing data from 242 patients admitted to the surgical intermediate and intensive care unit (88 community acquired and 154 postoperative peritonitis); they showed the importance of obtaining representativeness of pathogens responsible for IAI by Swab, taken from the intraperitoneal fluid immediately after laparotomy, of considering the polymicrobial nature of secondary peritonitis and the different resistance patterns of all pathogens identified to adapt common antimicrobial regimens and cover all relevant pathogens isolated; the principle of this approach is that the battleship is only sunk when all parts of it have been hit. ${ }^{13}$ The group reported that Enterococci were isolated in $47.1 \%$ of all patients, followed by Escherichia coli (42.6\%), other enterobacteriaceae (33.1\%), anaerobes (29.8\%) and Candida spp. (28.9\%). The susceptibility rates and spectrum adequacy rate (SAR) were lower in postoperative than in community acquired cases. The following regimens yielded a SAR $>95 \%$ when enterobacteriaceae only were considered: piperacillin/tazobactam + gentamicin, cefotaxim (only for community acquired cases), cefotaxim + gentamicin, meropenem, tigecycline + gentamicin or tigecycline + ciprofloxaxin. When enterococci were also considered, all betalactam based regimens required combination with vancomycin or linezolid for a SAR $>$ 95\%, whereas Tigecycline based regimens were not compromised. As for Candida spp., the SAR of fluconazole was $81.9-87.5 \% .^{13}$

This approach has its own logic in clinical practice, as empirical broad-spectrum antimicrobial treatment can expose patients to an overuse of antimicrobials, increasing AR.

Another effective clinical approach to limit the spreading of AR is the de-escalation strategy. It refers to the replacement of empirical broad-spectrum antimicrobial treatment with a narrower antimicrobial therapy, by either changing the pharmacological agent or discontinuing a pharmacological combination according to the patient's microbial culture results. ${ }^{15}$

It is known that patients with sepsis benefit from aggressive antimicrobial treatment in order to control high mortality rate reported in literature.

Turza et al. carried out a retrospective study to evaluate if antibiotic de-escalation strategy applied to critically ill patients was associated with increased patient mortality rates or worsening of the primary infection.

He concluded that antibiotic de-escalation was not associated with increased mortality rates, but the duration of antibiotic use was longer for patients treated in this way. Greater mortality rates were observed in the non-deescalated group, probably because the greater severity of disease classification (APACHE II). ${ }^{16}$

It is essential that the dosing of antibiotics is adequately adjusted in order to achieve an effective drug level above the minimum inhibition concentration but avoiding toxic side effects, above all in patients patients with acute kidney injury and continuous renal replacement therapy. ${ }^{17}$ Determination of serum levels should always be carried out, when this is possible. In any case, a sufficiently high loading dose should be included. ${ }^{17}$ Lower than standard dosages of renally excreted drugs must be administered in the presence of impaired renal function, while higher than standard dosages of renally excreted drugs may be required for optimal exposure in patients with glomerular hyperfiltration. ${ }^{7}$

In clinical practice all antibiotic treatment started with an intravenous administration followed by oral switch when appropriate (normal infection signs, normal infection laboratory parameters and resumption of oral feeding). ${ }^{18}$ Sartelli, after reviewing literature about duration of antibiotic therapy, concluded that in stable patients a short course of antimicrobial therapy (3-5 d) after adequate source control, depending on fever and leukocytosis, may be a reasonable option. In critically ill patients with severe sepsis and septic shock, an individualized approach is always mandatory and patient's inflammatory response should be monitored regularly by the dosage of Procalcitonin. ${ }^{19}$

\section{Antibiotic stewardship programs for surgeons}

For WHO guidelines, the rational use of drugs requires that patients receive medications appropriate to their clinical needs, in

Table 2. 2013 World Society Emergency Surgeons guidelines for management of intra-abdominal infections.

\begin{tabular}{|c|c|c|c|c|c|c|c|c|c|}
\hline \multicolumn{5}{|c|}{ Community acquired extra biliary IAIs } & \multicolumn{3}{|c|}{ Community acquired biliary IAIs } & \multicolumn{2}{|c|}{ Nosocomial extra biliary IAIs } \\
\hline $\begin{array}{c}\text { Patient Stable-non critical; } \\
\text { no risk factors } \\
\text { for ESBL }\end{array}$ & $\begin{array}{l}\text { Stable-non critical } \\
\text { with ESBL } \\
\text { associated risk } \\
\text { factors }\end{array}$ & $\begin{array}{l}\text { Critically ill with } \\
\text { no risk factors } \\
\text { for ESBL }\end{array}$ & $\begin{array}{l}\text { Critically ill } \\
\text { with ESBL } \\
\text { associated risk } \\
\text { factors }\end{array}$ & $\begin{array}{l}\text { Stable-non critical; } \\
\text { no risk factors } \\
\text { for ESBL }\end{array}$ & $\begin{array}{l}\text { Stable-non critical } \\
\text { with ESBL } \\
\text { associated risk } \\
\text { factors }\end{array}$ & $\begin{array}{l}\text { Critically ill with } \\
\text { no risk fac-tors } \\
\text { for ESBL } \\
\text { factors }\end{array}$ & $\begin{array}{l}\text { Critically ill } \\
\text { with ESBL } \\
\text { associated risk }\end{array}$ & $\begin{array}{c}\text { Stable-non critical; } \\
\text { risk for MDR } \\
\text { pathogens }\end{array}$ & $\begin{array}{l}\text { Critically ill; } \\
\text { risk for } \\
\text { MDR pathogens }\end{array}$ \\
\hline $\begin{array}{c}\text { Treatment Amoxicillin/ } \\
\text { clavulanate } \\
\text { or ciprofloxacin+ } \\
\text { metronidazole } \\
\text { in allergic patient }\end{array}$ & $\begin{array}{l}\text { Ertapenem } \\
\text { or tigecycline }\end{array}$ & $\begin{array}{l}\text { Piperacillin/ } \\
\text { tazobactam }\end{array}$ & $\begin{array}{l}\text { Meropenem or } \\
\text { imipenem } \\
\text { +/-fluconazole }\end{array}$ & $\begin{array}{l}\text { Amoxicillin/ } \\
\text { clavulanate or } \\
\text { ciprofloxacin } \\
\text { +metronidazole } \\
\text { in allergic patient }\end{array}$ & Tigecycline & $\begin{array}{l}\text { Piperacillin/ } \\
\text { tazobactam }\end{array}$ & $\begin{array}{l}\text { Piperacillin+ } \\
\text { tigecycline+/- } \\
\text { fluconazole }\end{array}$ & $\begin{array}{l}\text { Piperacillin+ } \\
\text { tigecycline+ } \\
\text { fluconazole }\end{array}$ & $\begin{array}{c}\text { Piperacillin+ } \\
\text { tigecycline+ } \\
\text { echinocandin } \\
\text { or meropenem, } \\
\text { imipenem, } \\
\text { teicoplanin+ } \\
\text { doripenem+ } \\
\text { echinocandin }\end{array}$ \\
\hline
\end{tabular}

IAl, intra-abdominal infection; ESBL, extended-spectrum beta-lactamase; MDR, multidrug resistant. 
doses that meet their own individual requirements, for an adequate period of time and at the lowest cost for themselves and their community. ${ }^{5}$ The research involved with the development of new antibiotics has not progressed in parallel with the increasing rate of AR, and clinicians do not have many options, which often are more expensive, to treat resistant infections. Adequate education programs and the application of evidence based international guidelines in clinical practice can decrease healthcare costs with considerable benefits in terms of cost effectiveness. De Simone et al., demonstrated in a retrospective study that an inexpensive and easy application of guidelines based on medical evidence, such as WSES guidelines for the management of IAI, in a department of Emergency and Trauma Surgery can lead to a significant reduction of hospital costs with improved outcomes. ${ }^{18}$

\section{Conclusions}

The use of antimicrobial agents is very high and often improper in the management of IAIs. $\mathrm{AR}$ is a direct consequence of the excessive and irrational use of antibiotics with limited therapeutic option in clinical practice because of the lack in the development of new and effective antimicrobial agents. Source control and empiric antibiotic therapy have to be administrated as early as possible to decrease high mortality rates in patients with severe sepsis or septic shock.

It is imperative for every surgeon to know that: prolonged peri-operative antibiotic prophylaxis is associated with an increased risk of Clostridium difficile-associated infection and side-effects (it does not reduce the number of wound infections); time to initiation of antibiotic treatment is the single strongest predictor of outcome; the principles of empiric antibiotic treatment should be defined according to the most frequently isolated germs, always taking into consideration the local trend of antibiotic resistance and the international evidencebased guidelines; the choice of the antimicrobial regimen depends on the source of intraabdominal infection, the risk factors for specific microorganisms, the resistance patterns and the clinical patient's condition (WSESSSS); the de-escalation strategy refers to the replacement of empirical broad-spectrum anti- microbial treatment with a narrower antimicrobial therapy, according to the patient's microbial culture results; it is effective in limiting the selection of resistant pathogens.

Proper antimicrobial stewardship in selecting an appropriate antibiotic and optimizing its dose and duration to cure IAIs may prevent the emergence of AR and decrease costs for antibiotics. A global cooperation to limit the spreading of AR is necessary.

\section{References}

1. Zhang YZ, Singh S.Antibiotic stewardship programmes in intensive care units: why, how, and where are they leading us.World J Crit Care Med 2015;4:13-28.

2. Ciorba V, Odone A, Veronesi L, et al. Antibiotic resistance as a major public health concern: epidemiology and economic impact. Ann Ig 2015;27:562-79.

3. Hagel S, Scheuerlein H. Perioperative antibiotic prophylaxis and antimicrobial therapy of intra-abdominal infections. Viszeralmedizin 2014;30:310-6.

4. Sartelli M, Catena F, Ansaloni L, et al. Complicated intra-abdominal infections worldwide: the definitive data of the CIAOW study. World J Emerg Surg 2014;9:37.

5. WHO global strategy for containment of antimicrobial resistance WHO/CDS/CSR/ 2001.2

6. European Centre for Disease Prevention and Control. Annual epidemiological report 2014. Antimicrobial resistance and healthcare-associated infections. Stockholm: ECDC; 2015.

7. Sartelli M, Viale P, Catena F, et al. 2013 WSES guidelines for management of intraabdominal infections. World J Emerg Surg 2013;8:3.

8. Sartelli M, Abu-Zidan FM, Catena F, et al. Global validation of the WSES Sepsis Severity Score for patients with complicated intra-abdominal infections: a prospective multicentre study (WISS Study).World J Emerg Surg 2015;10:61.

9. Zhang S, Huang W. Epidemiological study of community- and hospital-acquired intraabdominal infections. Chin $\mathrm{J}$ Traumatol 2015;18:84-9.

10. Solomkin JS, Mazuski JE, Bradley JS, et al.
Diagnosis and management of complicated intra-abdominal infection in adults and children:guidelines by the Surgical Infection Society and the Infectious Diseases Society of America. Surg Infect (Larchmt) 2010;11:79-109.

11. Sartelli M, Abu-Zidan FM, Ansaloni L, et al. The role of the open abdomen procedure in managing severe abdominal sepsis: WSES position paper. World J Emerg Surg 2015;10:35.

12. Sartelli M, Catena F, di Saverio S, et al. The challenge of antimicrobial resistance in managing intra-abdominal infections. Surg Infect (Larchmt) 2015;16:213-20.

13. Steinbach CL, Töpper C, Kees MG. Spectrum adequacy of antibiotic regimens for secondary peritonitis: a retrospective analysis in intermediate and intensive care unit patients. Ann Clin Microbiol Antimicrob 2015;14:48.

14. Membrilla-Fernández E, Sancho-Insenser JJ, Girvent-Montllor M, et al. Secondary peritonitis Spanish study group. Effect of initial empiric antibiotic therapy combined with control of the infection focus on the prognosis of patients with secondary peritonitis. Surg Infect (Larchmt) 2014;15:806-14.

15. Kaye KS. Antimicrobial de-escalation strategies in hospitalized patients with pneumonia, intra-abdominal infections, and bacteremia. J Hosp Med 2012;7 (Suppl.1):S13-21.

16. Turza KC, Politano AD, Rosenberger LH, et al. De-escalation of antibiotics does not increase mortality in critically ill surgical patients. Surg Infect (Larchmt) 2016;17:48-52.

17. Michael E, Kindgen-Milles D. Antibiotic dosing for renal function disorders and continuous renal replacement therapy. Anaesthesist 2015;64:315-23.

18. De Simone B, Coccolini F, Catena F, et al. Benefits of WSES guidelines application for the management of intra-abdominal infections. World J Emerg Surg 2015;10:18.

19. Sartelli M, Catena F, Ansaloni L, et al. Duration of antimicrobial therapy in treating complicated intra-abdominal infections: a comprehensive review. Surg Infect (Larchmt) 2016;17:9-12. 